\title{
Colonization and infection with a rare microorganism in a neonatal intensive care unit: three preterm infants with Elizabethkingia meningoseptica
}

\author{
Kiymet Celika, M.D., Demet Terek", M.D., Ozgur Olukman ${ }^{a}$,Associate Professor, Gamze Gulfidan ${ }^{b}$, M.D., \\ Sebnem Calkavura, M.D. Associate Professor, Ilker Devrimc , M.D. Associate Professor and \\ Sertac Arslanoglua, M.D. Professor
}

\begin{abstract}
Elizabethkingia meningoseptica is a widespread gram-negative bacillus in the environment, but a rarely reported human pathogen presenting mostly as nosocomial infections. Advances in neonatal intensive care facilities and usage of sophisticated medical devices strengthen the invasive infectious potential of the microorganism. Clinical manifestations usually include primary bacteremia, meningitis, nosocomial pneumoniae, intravascular catheter-related bacteremia and gastrointestinal and biliary tract infections. Lack of improved diagnostic systems in resource constrained settings, might be a cause of underreporting of such infections. Discrimination between colonization and infection is quite difficult, and it has an unusual antibiotic susceptibility pattern. Therefore clinicians should pay special attention to accurate diagnosis in order to prevent mistreatment. Here we report three newborn cases with the diagnosis of E. meningoseptica infection and colopnization, with the aim of drawing attention to the diagnosis and management of this rare but lethal bacteria that is already present in the intensive care unit environment.
\end{abstract}

Key words: Elizabethkingia meningoseptica, nosocomial infection, infant newborn.

http: / / dx.doi.org/10.5546/ aap.2019.eng.e631

To cite: Celik K, Terek D, Olukman O, Gulfidan G, et al. Colonization and infection with a rare microorganism in a neonatal intensive care unit: three preterm infants with Elizabethkingia meningoseptica. Arch Argent Pediatr 2019;117(6):e631-e634.
a. Division of Neonatology.
b. Microbiology Laboratory.
c. Division of Pediatric Infectious Diseases.
Dr. Behcet Uz Children's Hospital, Izmir, Turkey.

E-mail address:

Kiymet Celik, M.D.: kiymetcelikmd@gmail.com

Funding: None.

Conflict of interest: None.

Received: 7-24-2018

Accepted: 6-6-2019

\section{INTRODUCTION}

The gram negative bacillus Elizabethkingia meningoseptica (E. meningoseptica) is widely distributed in nature, mostly in plants, soil and water sources. It may also stay alive in chlorine-treated water supplies and hospital environment. ${ }^{1}$ Although known as a low-virulence bacteria, it can cause rare but severe human infections, especially in immunocompromised hosts including newborn infants via colonized indwelling devices such as intubation tubes, humidifiers, respirator circuits and newborn incubators. ${ }^{1,2}$ E. meningoseptica infection in neonates, usually has a serious clinical course with a fatal outcome or with severe neurologic impairment in survivors. ${ }^{3}$ Here we report ourexperience of three newborn cases with E. meningoseptica, to emphasize the importance of clinical course, management and differential diagnosis between actual infection and colonization.

\section{CASE REPORTS \\ Case 1}

A 26 gestational weeks, 1000 grams male infant born to a 23-year-old mother was referred to our neonatal intensive care unit (NICU) on day 26, with the diagnosis of persistent patent ductus arteriosus (PDA) despite medical treatment with oral paracetamol. Because of cardiorespiratory failure at admission, the infant was intubated and started on mechanical ventilation. Two courses of intravenous ibuprofen was administered and PDA was successfully closed without need for surgical treatment. On the $28^{\text {th }}$ day of hospitalization, while still on mechanical ventilation, his clinical status deteriorated requiring further ventilator adjustments. Laboratory tests revealed increased acute phase reactants and leucocytosis with increased neutrophil counts. The chest X-ray revealed bilateral pulmonary infiltrations. Blood, vascular catheter, urine and cerebrospinal fluid (CSF) cultures were obtained. Bronchoalveolar lavage samples were also obtained with the 
suspicion of ventilatory associated pneumonia. E. Meningoseptica, susceptible to ciprofloxacin and cefoperazone-sulbactam, was isolated. However other cultures were all negative for $E$. meningoseptica. Diagnosis of ventilator-associated pneumonia (VAP) was made according to the diagnostic criteria of Centers for Disease Control and Prevention (CDC). The infant responded to $100 \mathrm{mg} / \mathrm{kg}$ intravenous cefoperazone-sulbactam twice a day administered for fourteen days.

The clinical condition improved gradually, being extubated at the $56^{\text {th }}$ day of hospital stay. He was discharged with full recovery on the $91^{\text {th }}$ day of life.

\section{Case 2}

A 28 gestational weeks, 1180 grams male infant, twin-born to a 33-year-old mother was referred to our NICU within the first hour of life, after being intubated in the delivery room. He received endotracheal surfactant for respiratory distress syndrome (RDS) and intravenous ibuprofen for PDA. On follow-up, he developed grade 3 intraventricular hemorrhage (IVH). He was extubated on day 16 . However, his clinical condition deteriorated on day 21, developing abdominal distention and respiratory distress resulting in re-intubation. Late-onset nosocomial neonatal sepsis was diagnosed and after obtaining blood, urine, cerebrospinal fluid (CSF) and tracheal aspirate cultures, empirical vancomycin and piperacillin-tazobactam treatment was started. Tracheal aspirate culture revealed E. meningoseptica susceptible to ciprofloxacin. Since there was no worsening in the chest $X$-ray, and the infant responded to empirical antimicrobial therapy, the isolation was accepted as colonization and no treatment change was implemented. Control tracheal aspirate cultures were negative for any bacterial growth. He was extubated on day 26 and discharged on day 110 with some other complications due to prematurity.

\section{Case 3}

A 27 gestational weeks, 1100 grams female infant, born to a 26-year-old mother as a triplet was referred to our NICU on the $5^{\text {th }}$ hour of life with the diagnosis of RDS. She was intubated and endotracheal surfactan was administered. She also had an accompanying PDA presenting with severe hypotension, metabolic acidosis, as well as thrombocytopenia and bilateral grade 3 IVH. After completing her treatment against above-mentioned clinical conditions, she was stabilized and had an uneventful follow up for the next four weeks. Unfortunately her clinical condition worsened on day 30 and she developed hypotonia, apnea and mixed acidosis. With the suspicion of late-onset nosocomial neonatal sepsis, she was put on empirical vancomycin, meropenem and amikacin treatment after obtaining blood, urine and cerebrospinal fluid (CSF) cultures. She was immediately intubated and put on mechanical ventilation due to respiratory failure. On day 35 , her respiratory secretions became purulent and her chest X-ray revealed ground glass appearance associated with pneumonia. Tracheal aspirate culture was obtained revealing growth for E. meningoseptica susceptible to ciprofloxacin and levofloxacin. Other cultures were all negative for any bacterial growth. Ciprofloxacin treatment was initiated. Her clinical status recovered gradually, however her serial tracheal aspirate cultures remained persistently positive for E. meningoseptica for nearly two weeks. Ongoing cultures were accepted as colonization since no any further worsening in her clinical status was observed on follow-up. She was extubated on day 16 and ciprofloxacin treatment was completed to 21 days. She was discharged on day 147 with several comorbidities related to prematurity.

\section{DISCUSSION}

Gram-negative bacterial infections are one of the main problems of NICUs and the infected hands of the staff has been reported to be the most important factor for transmission within the unit. ${ }^{4,8}$ Most Gram-negative bacteria may survive in the hospital environment by colonizing on the walls, surfaces of the furniture, gadgets, medical equipments, various devices and instruments. Colonization may also be on the skin and mucosal surfaces of both the staff and the patients. Once colonized in human beings, the bacteria may stay harmless or may cause invasive infections such as primary bacteremia, VAP, intravascular catheter-related bacteremia or gastrointestinal tract infections. ${ }^{8}$

E. meningoseptica is a ubiquitous, waterborne, saprophytic bacillus which is not considered to be a part of the normal human flora. It has a low degree of pathogenicity and only a small percentage of colonized patients develop sepsis, especially the ones who are immunocompromised. ${ }^{5}$ In the current literature, intermittent epidemics in NICUs and nurseries 
have been reported ${ }^{4}$. In many environmental studies, it is demonstrated that this organism can survive in moist such as ventilator tubes, tap water supplies of hospitals, aspiration fluids and oxygen humidifiers. ${ }^{2}$ Person-to-person spread is unusual, as manifested by the low rates of infection among neonates. In the study of Gungor et al., researchers stated that they had isolated E. meningoseptica from total parenteral nutrition fluids. ${ }^{1}$

In an immunocompetent host, E. meningoseptica is cleared rapidly by immune defenses without antibiotic treatment. However, due to their weak immune response preterm infants are the most susceptible group for E. meningoseptica infection. ${ }^{5}$ In the series of Issack et al., seven of eight $E$. meningoseptica neonatal meningitis cases had a birth weight of $<2,500$ grams and the mean age at the time of diagnosis was 10 days. ${ }^{6}$ All of our cases were $\leq 28$ gestational weeks and $<1,500$ grams at birth.

In our experience, we saw the two possible forms of bacterial isolation, both infection and colonization, for E. meningoseptica. In the first and third cases, VAP was the major clinical presentation diagnosed with positive bronchoalveolar lavage and tracheal aspirate cultures. In the third case, the clinical course was rather severe requiring fluoroquinolone treatment. On the other hand, isolation of E. meningoseptica from tracheal aspirate culture was accepted as colonization in the second case, due to lack of respiratory symptoms and normal chest X-ray. With regard to our limited experience in three cases, we conclude that, it is important to make the precise differential diagnosis between actual infection and colonization to avoid unnecessary antibiotic treatment and treatment related costs and complications. Thus our second case, did not receive any other treatment than standard late-onset neonatal sepsis protocols. E. meningoseptica colonization in neonates does not necessarily lead to infection and that such colonization outbreaks may be controlled with emphasis on the standard precautions. ${ }^{9}$

The agent is well known to be resistant to most antibiotics and the inappropriate use of empirical antibiotics may contribute to poor clinical outcome. Susceptibility test results may vary according to the preferred study method. Due to the production of two beta lactamases, one extended spectrum betalactamase (ESBL) and one class B carbapenem-hydrolyzing metallolactamase, many E. meningoseptica strains are usually resistant to extendedspectrum beta-lactam antibiotics including carbapenems and aztreonam. ${ }^{7}$ E.meningoseptica is also resistant to aminoglycosides, chloramphenicol and erythromycin, while in vitro, however, fluoroquinolones, trimethoprim/ sulfamethoxazole and piperacillin/tazobactam are reported to be effective against this pathogen. ${ }^{7,10}$ We observed that the strains isolated from all three cases were resistant to ampicillin, gentamycin and meropenem, while on the other hand sensitive to ciprofloxacin.

In conclusion, isolation of E. meningoseptica from tracheal aspirate cultures does not always point to E. meningoseptica as the real cause of infection. It may only be interpreted as colonization with respect to favorable clinical status and relatively normal laboratory findings. Therefore in this present case report, we would like to emphasize the importance of differentiating infection from colonization in order to avoid unnecessary antibiotic treatment and treatment related undesirable complications, as well as high treatment costs.

\section{REFERENCES}

1. Güngör S, Ozen M, Akinci A, Durmaz R. A Chryseobacterium meningoseptica outbreak in a neonatal ward. Infect Control Hosp Epidemiol. 2003; 24(8):613-7.

2. Kirby JT, Sader HS, Wlash TR, Jones RN. Antimicrobial susceptibility and epidemiology of a wolrdwide collection of Chryseobacterium spp: report form the SENTRY Antimicrobial Surveillance Program (1997-2001). JClin Microbiol.2004;42(1):4458.

3. Tai IC, Liu TP, Chen YJ, Lien RI, et al. Outbreak of Elizabethkingia meningoseptica sepsis with meningitis in a well-baby nursery. J Hosp Infect. 2017; 96(2):16871.

4. Hoque SN, Graham J, Kaufmann ME, Tabagchali S. Chryseobacterium (Flavobacterium) meningoseptica outbreak associate with colonization of water taps in a neonatal intensive care unit. J Hosp Infect. 2001; 47(3):188-92.

5. Bloch KC, Nadarajah R, Jacobs R. Chryseobacterium meningoseptica: An emerging pathogen among immunocompromised adults: Report of 6 cases and literature review. Medicine (Baltimore). 1997;76(1):3041.

6. Issack MI, Neetoo Y. An outbreak of Elizabethkingia meningoseptica neonatal meningitis in Mauritus. J Infect Dev Ctries. 2011; 5(12):834-9.

7. Amer MZ, Bandey M, Bukhari A, Nemenqani D. Neonatal meningitis caused by Elizabethkingia meningoseptica in Saudi Arabia. I Infect Dev Ctries. 2011; 5(10):745-7. 
8. YoonHS. TwocasesofChryseobacteriummeningoseptica infection in neonatal intensive care unit. Koren J Pediatr. 2007; 50(7):698-701.

9. Maraki S, Scoulica E, Manoura A, Papageorgiou N, et al. A Chryseobacterium meningoseptica colonization outbreak in a neonatal intensive care unit. Eur J Clin Microbiol Infect Dis. 2009; 28(12):1415-9.
10. Mirza HC, Tuncer Ö, Ölmez S, Şener B, et al. Clinical Strains of Chryseobacterium and Elizabethkingia spp. Isolated from Pediatric Patients in a University Hospital: Performance of MALDI-TOF MS-Based Identification, Antimicrobial Susceptibilities, and Baseline Patient Characteristics. Microb Drug Resist. 2018; 24(6):816-21. 